\title{
Effects of modeling liquid/resin and polishing on the color change of resin composite
}

\begin{abstract}
José Augusto SEDREZ-PORTO(a) Eliseu Aldrighi MÜNCHOW(a) Lucas Pradebon BRONDANI(a) Maximiliano Sergio CENCI(a) Tatiana PEREIRA-CENCI (a)
\end{abstract}

(a) Universidade Federal de Pelotas, School of Dentistry, Graduate Program in Dentistry, Pelotas, RS, Brazil.

Declaration of Interests: The authors certify that they have no commercial or associative interest that represents a conflict of interest in connection with the manuscript.

Corresponding Author:

Eliseu Aldrighi Münchow

E-mail: eliseumunchow@gmail.com

Submitted: Dec 02, 2015

Accepted for publication: Apr 25, 2016

Last revision: May 05, 2016

\begin{abstract}
Modeling liquids/resins have been used to build up resin composite $(\mathrm{RC})$ restorations, although there is a lack of information regarding their effects on the color stability of the latter. Therefore, the purpose of the present study was to evaluate the effects of the presence of modeling liquid between layers of RC and the finishing/polishing state of the material on color change in specimens exposed to red wine staining over time. Specimens were prepared by placing four increments $\left( \pm 0.5 \mathrm{~mm}\right.$ thick) of RC (Filtek ${ }^{\mathrm{TM}} \mathrm{Z} 350 \mathrm{XT}, 3 \mathrm{M}$ ESPE) into molds; half of which were prepared by applying modeling liquid (Scotchbond $^{\mathrm{TM}}$ Multi-Purpose ${ }^{\mathrm{TM}}$ Adhesive, SBMP, 3M ESPE) between the layers of $\mathrm{RC}$, whereas the other half were prepared without SBMP (control). Light-activation was performed after application of the final RC layer using a light-emitting diode (Radii, SDI) curing unit with an irradiance of $900 \mathrm{~mW} / \mathrm{cm}^{2}$ for $20 \mathrm{~s}$. Each group was divided according to the surface finishing protocol $(n=7)$ : nothing (non-polished) or polishing with Sof-Lex ${ }^{\mathrm{TM}} /$ diamond paste (polished). Initial colors of the specimens were evaluated with a digital spectrophotometer and the CIEL ${ }^{*} a^{*} b^{*}$ color system. The specimens were stored in wine $\left(37^{\circ} \mathrm{C}\right)$ for 12 months, and the color measurements were reassessed after 4, 6, and 12 months of storage. Scanning electron microscopy (SEM) analysis was performed at the end. Data were analyzed using ANOVA and Tukey's test $(\alpha=5 \%)$. The presence of SBMP resulted in lower overall color change of the RC as compared with the control. The non-polished specimens exhibited a significantly higher color change than the polished specimens. SEM images corroborated the previous findings. In summary, the use of modeling liquid between layers of RC shows potential for application to reduce or delay the staining process of RC over time. Moreover, polishing is essential to provide increased color stability of the RC restoration.
\end{abstract}

Keywords: Aging; Dental Materials; Microscopy, Electron, Scanning.

\section{Introduction}

Resin composites (RCs) are presently used for direct and indirect dental restoration purposes due to their satisfactory physical and mechanical properties, as well as their excellent esthetic characteristics. However, a proper layering technique during composite insertion is essential to obtain these beneficial characteristics, mainly due to the following two 
reasons: firstly, composites have a limited depth of cure, which may compromise light transmission through the material and consequent polymerization if thick layers (e.g., $>2 \mathrm{~mm}$ ) are used. ${ }^{1,2}$ Secondly, to obtain an outstanding and undetectable restoration, a combination of different composites of different shades should be used (i.e., the stratification technique). Therefore, the application of several layers of material is a common clinical step during the sculpting process of composite restorations. ${ }^{3,4}$

One of the main drawbacks of sculpting composite restorations is that some materials are sticky, reducing the ease of handling and insertion of the material into the tooth cavity. Some materials readily adhere to the composite instruments; thus, limiting reestablishment of the shape and anatomical contour of the tooth. As a result, the application of lubricants on the composite instrument, including isopropyl alcohol and acetone, ${ }^{5,6,7}$ or of modeling liquids/resins, including adhesive resins, between the layers of composite ${ }^{5}$ have been proposed. This technique increases the ease of insertion of composites, improving both the adaptation to the tooth cavity and the modeling of the material. ${ }^{8}$ Although the use of modeling liquids may enhance the handling application of composites, there are few reports in the literature to support this technique. Moreover, and according to Tuncer et al., ${ }^{9}$ who investigated the hardness, roughness, and color stability of composite specimens that received a superficial layer of resin adhesive as a modeling resin, the modeling resin may negatively affect some of these properties, although this was dependent on the type of composite tested.

Color is one of the most important characteristics of composite restorations. However, obtaining a perfect match between composite shades and the color appearance of the natural tooth is challenging to achieve, leading to patient dissatisfaction. ${ }^{10}$ Furthermore, change in the color of the restorative material is a frequent consequence of composite restorations over time, which can be influenced by several factors, including the composition of the material (i.e., the ratio between organic matrix and filler particles), ${ }^{11}$ the type of staining media/environment, ${ }^{12,13}$ and the polishing state of the surface of the material. ${ }^{14}$ In considering the use of modeling liquids for sculpting
$\mathrm{RC}$ restorations, the presence of the modeling liquid between the layers of composites may, at least in theory, change the translucency and physical stability of the material, altering color stability over time. On the other hand, proper finishing and polishing procedures could be used to overcome this problem. ${ }^{9}$ Taking these factors into consideration, the present study evaluated the effects of the presence of a modeling liquid (adhesive resin) between layers of RC and the finishing/polishing state of the material on the change in the color of specimens exposed to red wine staining over time. The hypotheses were (1) the presence of a modeling liquid within the composite structure would increase the color change over time and (2) the smoother the surface of the material, the lower the change in color.

\section{Methodology}

\section{Study design and specimen preparation}

The present study was designed to investigate the effects of three independent variables, "presence of modeling liquid," "polishing state," and "storage period," on the change in the color of RC specimens. Thus, the specimens were prepared with or without the modeling liquid, were either polished or remained unpolished, and were stored in red wine for 12 months. The specimens were analyzed at the following time points: baseline, and after 4, 6, and 12 months of storage. Filtek ${ }^{\mathrm{TM}}$ Z350 XT (3M ESPE, St. Paul, USA) was used as the composite material and Adper ${ }^{\mathrm{TM}}$ Scotchbond ${ }^{\mathrm{TM}}$ Multi-Purpose Adhesive (3M ESPE) was used as the modeling liquid.

A total of 28 disc-shaped specimens $(6 \mathrm{~mm}$ diameter $\times 2 \mathrm{~mm}$ thickness) were prepared by placing four increments of composite into silicone molds. Half of the specimens were prepared without applying the modeling liquid (control group), whereas the remaining half (SBMP group) was prepared by applying one increment of composite (approximately $0.5 \mathrm{~mm}$ thick), followed by one layer of modeling liquid; this composite and modeling liquid application was repeated twice, resulting in the total application of four increments of composite and three layers of the modeling liquid, simulating an incremental restoration similar to that usually performed in the anterior 
teeth. After placing the materials, the specimens were light-activated for $20 \mathrm{~s}$ at both the top and bottom surfaces using a light-emitting diode (LED) light-curing unit (Radii, SDI, Bayswater, Australia), which was continuously monitored to ensure that $900 \mathrm{~mW} / \mathrm{cm}^{2}$ of irradiance was applied to each specimen. Each group of specimens was then divided into two subgroups according to the surface finishing treatment applied $(n=7)$ : no treatment (non-polished group) or finishing/polishing (polished group) using a complete sequence of Sof-Lex polishing discs (Pop On Orange series, 3M ESPE), from medium to extra-fine ${ }^{15}$ for $15 \mathrm{~s}$ each, followed by the application of felt discs (Diamond flex 12 mm, FGM, Joinville, Brazil) and diamond paste (Diamond AC II, FGM). The polishing procedure was performed by holding the specimen with tweezers.

\section{Color evaluation and staining of specimens}

The initial (baseline) color of all specimens was evaluated using a digital spectrophotometer (Vita Easyshade, Vita Zahnfabrik, Bad Sackingen, Germany) and the CIEL $L^{*} a^{*} b^{*}$ color system. The specimens were measured against a white Plexiglass background to eliminate background light, and the $L^{*}$ (white/black), $a^{*}$ (red/green), and $b^{*}$ (yellow/blue) color parameters were obtained. Subsequently, all specimens were placed into wells of a 24-well plate and immersed in red wine (Concha y Toro, Cabernet Sauvignon 2007, Las Condes, Chile; pH 3.6; 14.5\% alcohol by volume) for 12 months. The wine was replaced weekly. After 4, 6, and 12 months of storage, each specimen was washed in distilled water, air-dried, and measured again for color reassessment. The color change after each time point was calculated, as previously described ${ }^{16}$, using the following formula: $\Delta E^{*}=\left[\left(\Delta L^{*}\right)^{2}+\left(\Delta a^{*}\right)^{2}+\left(\Delta b^{*}\right)^{2}\right]^{1 / 2}$, where $\Delta L^{*}, \Delta a^{*}$, and $\Delta b^{*}$ are the difference between the final and initial $L^{*}, a^{*}$, and $b^{*}$ color parameters, respectively.

\section{Scanning electron microscopy (SEM) analysis}

After 12 months of wine storage, two specimens from each group were selected for SEM evaluation. One of the specimens remained unmodified for analysis of the surface topography, whereas the other specimen was divided into two halves in order to analyze the cross-sectional surface (interface) of the specimen. Two fresh composite specimens, which were not exposed to wine storage or modeling liquid application were also prepared for comparative purposes; these specimens were polished using the same finishing/polishing protocol performed using the other specimens. All samples were dried in a desiccator at $37^{\circ} \mathrm{C}$, sputter-coated with gold/palladium, and then evaluated under SEM (SSX-550, Shimadzu, Tokyo, Japan). The images obtained were qualitatively analyzed.

\section{Statistical analysis}

All data were analyzed using the statistical program, SigmaPlot 12 (Systat Software Inc., San Jose, USA). Two-way repeated measures analysis of variance and Tukey's test were used to analyze the effect of the "presence of modeling liquid" and "storage period" on the $\Delta \mathrm{E}^{*}$ of the polished and non-polished specimens; whereas t-tests were used to analyze the effect of the "polishing state" on the $\Delta \mathrm{E}^{*}$ of groups at each time point investigated. All analyses were set at a $5 \%$ level of significance.

\section{Results}

\section{Color change}

The statistical analysis revealed that there was a statistically significant interaction between the factors investigated $(\mathrm{p} \leq 0.015)$. Figure 1 illustrates the changes obtained using the $L^{*}, a^{*}$, and $b^{*}$ color parameters at each time point. After 12 months of wine storage, the non-polished specimens displayed considerably lower lightness (decrease in the $L^{*}$ axis; Figure 1a), higher redness (increase in the $a^{*}$ axis; Figure 1b), and higher yellowness (increase in the $b^{*}$ axis; Figure 1c), compared with the polished specimens. In addition, in the SBMP group, the overall change in the color parameters was lower as compared with that in the control group. Regarding the overall color change $\left(\Delta \mathrm{E}^{*}\right)$ of the specimens (Figure $1 \mathrm{~d}$ and Table 1), the control group exhibited higher $\Delta \mathrm{E}^{*}$ values, compared with the SBMP group $(\mathrm{p}<0.001)$, which was irrespective of the polishing state of the specimens. The only exception was observed with the polished specimens after 4 months of wine storage, 
which exihibited similar color change ( $p=0.597)$. Moreover, the duration of the storage period had no effect on the color change of the specimens within the control group ( $p \geq 0.164)$; whereas, after 12 months of wine storage, the polished and non-polished SBMP specimens exhibited lower $(\mathrm{p}<0.001)$ and higher $(\mathrm{p}=0.038) \Delta \mathrm{E}^{*}$ values, respectively, compared with the values after 4 months (Table 1 ).

Regarding the effect of the "polishing state" on the color change of the specimens, the non-polished specimens exhibited significantly higher $\Delta \mathrm{E}^{*}$ values, compared with the polished specimens ( $\mathrm{p} \leq 0.003)$, regardless of the duration of the storage period, with the exception of the SBMP group after 4 months of wine storage (Table 1), in which the polished specimens exhibited similar $\Delta \mathrm{E}^{*}$ values to those in the non-polished specimens $(p=0.055)$.

\section{SEM analysis}

Figure 2 shows SEM images of the topography and cross-sectional surface of a polished composite specimen, which was not subjected to wine storage (fresh composite). The images show that the specimen was smooth and without defects, with the presence of scratches only on the surface (Figure 2a,b). In addition, the cross-sectional surface was uniform with no different material (i.e., modeling liquid) detected inside (Figure 2c,d). In the wine-stored specimens, the topography and cross-sectional surface after 12 months of storage can be seen in Figure 3. According to the SEM images, clear differences between the groups were observed: first, the non-polished specimens exhibited rougher surfaces, compared with their polished counterparts, with the occurrence of a crust complex, which was extensively distributed over the surface; second, the SBMP group exhibited a considerably smoother surface, compared with the control group in the polished specimens, whereas the non-polished specimens in both the SBMP and control groups exhibited a non-uniform surface; however, the crust complex within the control group appeared thicker than that in the SBMP group. The cross-sectional surface of the control group was similar to that of the fresh composite group (Figure 2c,d), whereas a heterogeneous cross-sectional surface was observed in the SBMP group, with a different phase/material clearly identified within the composite structure.

\section{Discussion}

The ease of handling of $\mathrm{RC}$ restorations resulting from the use of modeling liquids has led to several dental professionals using this technique. However, doubts have arisen regarding possible changes in the characteristics and properties of composites caused by the application of modeling liquids, particularly those related to the color stability of the restoration over time. ${ }^{9}$ Thus, the present study evaluated the effects of the presence of modeling liquid and polishing state on the color change of RC exposed to red wine staining over time. On analysis of the presence of modeling liquid, the specimens prepared with modeling liquid exhibited reduced overall color change, compared with the specimens prepared without modeling liquid, thus rejecting the first hypothesis of the study.

It is well known that color change in RCs is directly influenced by both intrinsic and extrinsic factors. While the former relates to the chemical composition, including the type of organic matrix, filler particles and photo-initiators, ${ }^{11}$ and the polymerization state of the material; ${ }^{17}$ the latter is related to the interaction of staining substances, including food colorants and beverages, with the composite structure. ${ }^{18}$

Table 1. Color change $\left(\Delta E^{*}\right.$, mean \pm standard deviation) of the polished and non-polished specimens after immersion in red wine at different time points.

\begin{tabular}{|c|c|c|c|c|c|c|}
\hline \multirow{2}{*}{ Group } & \multicolumn{3}{|c|}{ Polished specimens } & \multicolumn{3}{|c|}{ Non-polished specimens } \\
\hline & 4 months & 6 months & 12 months & 4 months & 6 months & 12 months \\
\hline Control & ${ }^{A} 22.0( \pm 3.4)^{a}$ & ${ }^{A} 21.1( \pm 2.6)^{a}$ & ${ }^{A} 24.1( \pm 3.1)^{\circ}$ & A $47.2( \pm 1.6)^{\circ}$ & A $47.7( \pm 1.0)^{a}$ & ${ }^{A} 50.8( \pm 2.0)^{\circ}$ \\
\hline SBMP & ${ }^{A} 23.0( \pm 3.2)^{a^{*}}$ & ${ }^{\text {в }} 13.9( \pm 2.6)^{b}$ & в $15.6( \pm 3.1)^{b}$ & 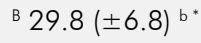 & ${ }^{\text {B }} 23.1( \pm 4.9)^{\mathrm{C}}$ & ${ }^{\text {в }} 35.5( \pm 9.6)^{\text {व }}$ \\
\hline
\end{tabular}

Distinct uppercase letters in the same column and lowercase letters in the same row indicate, respectively, statistically significant differences among groups $(p<0.05)$. "Groups typed with this symbol demonstrated similar color change results, regardless of the polishing state of the material $(p>0.05)$. 
In the present study, composite specimens were stored in red wine for a 12-month period, which was performed to simulate the marked staining that composite restorations may be exposed to over time. ${ }^{19}$ The specimens prepared with the modeling liquid exhibited considerably less discoloration, compared with the specimens prepared without modeling liquid, and this may be explained by the fact that the modeling liquid used, a hydrophobic resin, might have enhanced the cohesion between the composite increments, thus improving the chemo-physical stability of the material; this has been confirmed in a recent study. ${ }^{20}$ Consequently, the hydrolysis of the material was limited, leading to lower surface staining. Of note, after 4 months of wine storage, the presence of modeling liquid had no beneficial effect on the color stability of the RC, although this was only observed if the material was polished (Figure $1 \mathrm{~d}$ and Table 1). The staining of the composite restorations has been revealed to be a time-dependent process, ${ }^{21}$ and it may
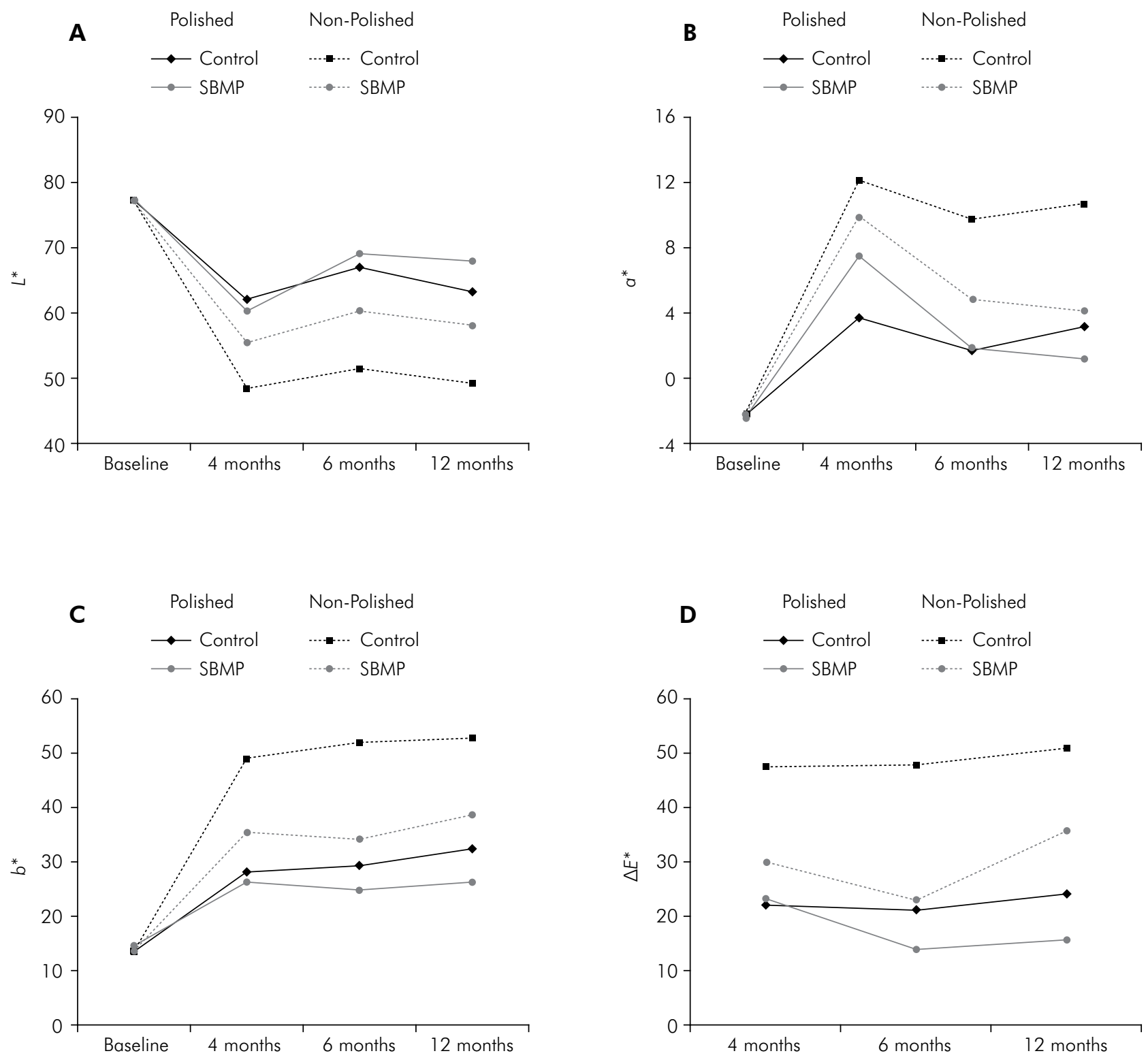

Figure 1. Color parameters tested in the study for the polished and non-polished specimens, with (SBMP) or without (control) application of modeling liquid: $L^{*}(a), a^{*}(b), b^{*}(c)$, and color change $/ \Delta E^{*}(d)$. 

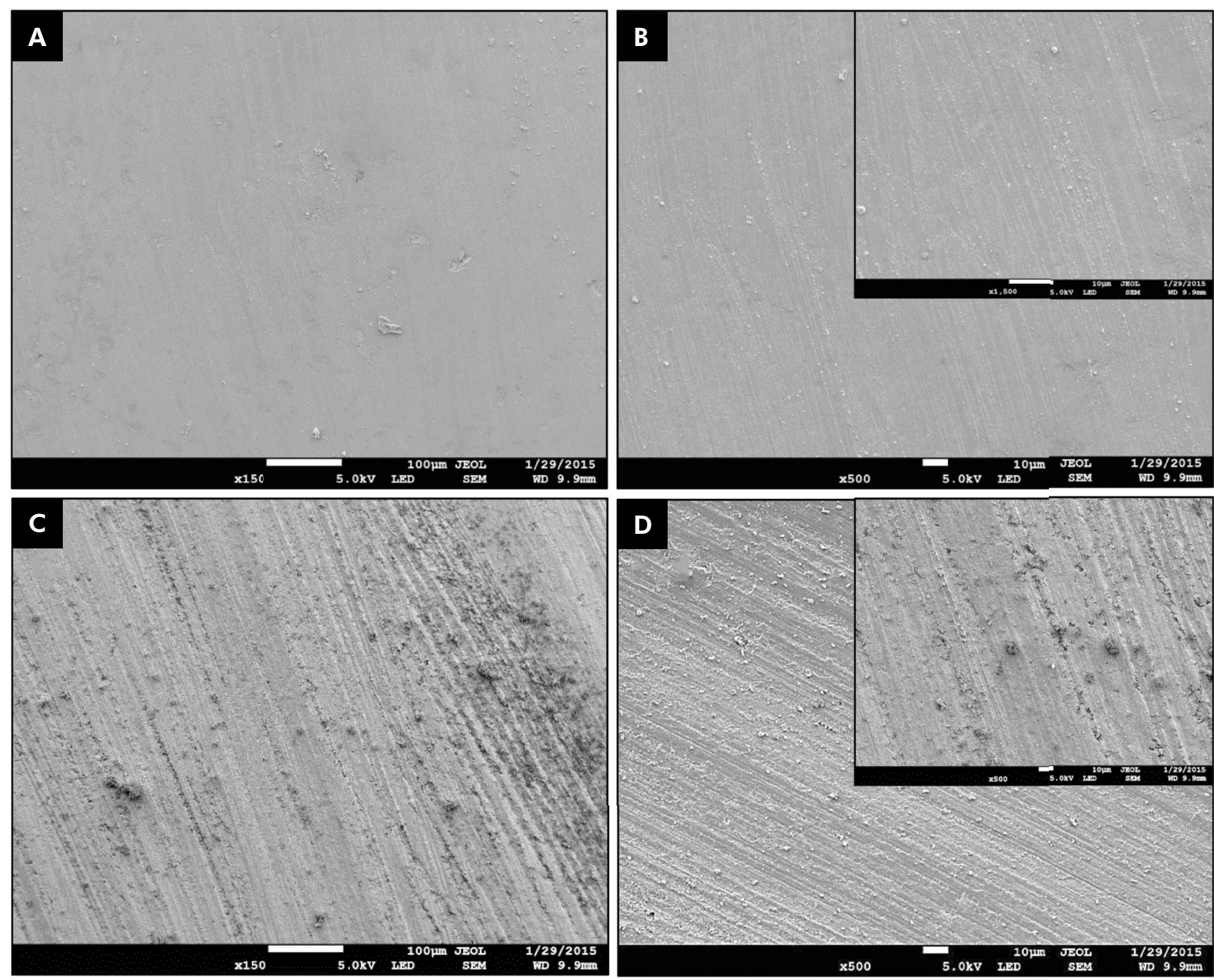

Figure 2. SEM images showing the surface topography (images " $a$ " and " $b$ ") and cross-sectional surface (images " $c$ " and " $d$ ") of a composite resin specimen without storage in red wine. Images " $a$ " and " $c$ " represent 150× magnification, and images " $b$ " and " $d$ " 500× magnification; inchart images in " $b$ " and " $d$ " represent $1.500 \times$ magnification.

be that a duration of 4 months is too short to detect significant differences between two distinct treatment modalities. After 6 months of wine storage, significant differences were observed between the specimens with and without modeling liquid, regardless of the polishing state of the material. The presence of modeling liquid acted as a protective agent against severe discoloration of the composite.

The positive effect of the SBMP adhesive resin used in the present study as a modeling liquid may be explained by the ability to form stable intermolecular chains with the monomeric units of the $\mathrm{RC}$, reducing the penetration of the wine staining/molecules on the surface and inner structure of the material. According to Barcellos et al., ${ }^{8}$ SBMP adhesive improved the strength of the restorations, corroborating with the present findings and confirming that this adhesive resin serves as a good modeling liquid for RCs.

In terms of polishing state, it was observed that the polished specimens exhibited less color change, compared with the non-polished specimens, which confirm the second hypothesis of the study. There are several previous reports stating that a polished surface is less susceptible to staining:, ${ }^{14,22,23}$ indeed, the proper finishing and polishing of a restoration may be beneficial to the maintenance of esthetics and glossy characteristics. ${ }^{24,25}$ Moreover, the polishing of composites is usually performed 


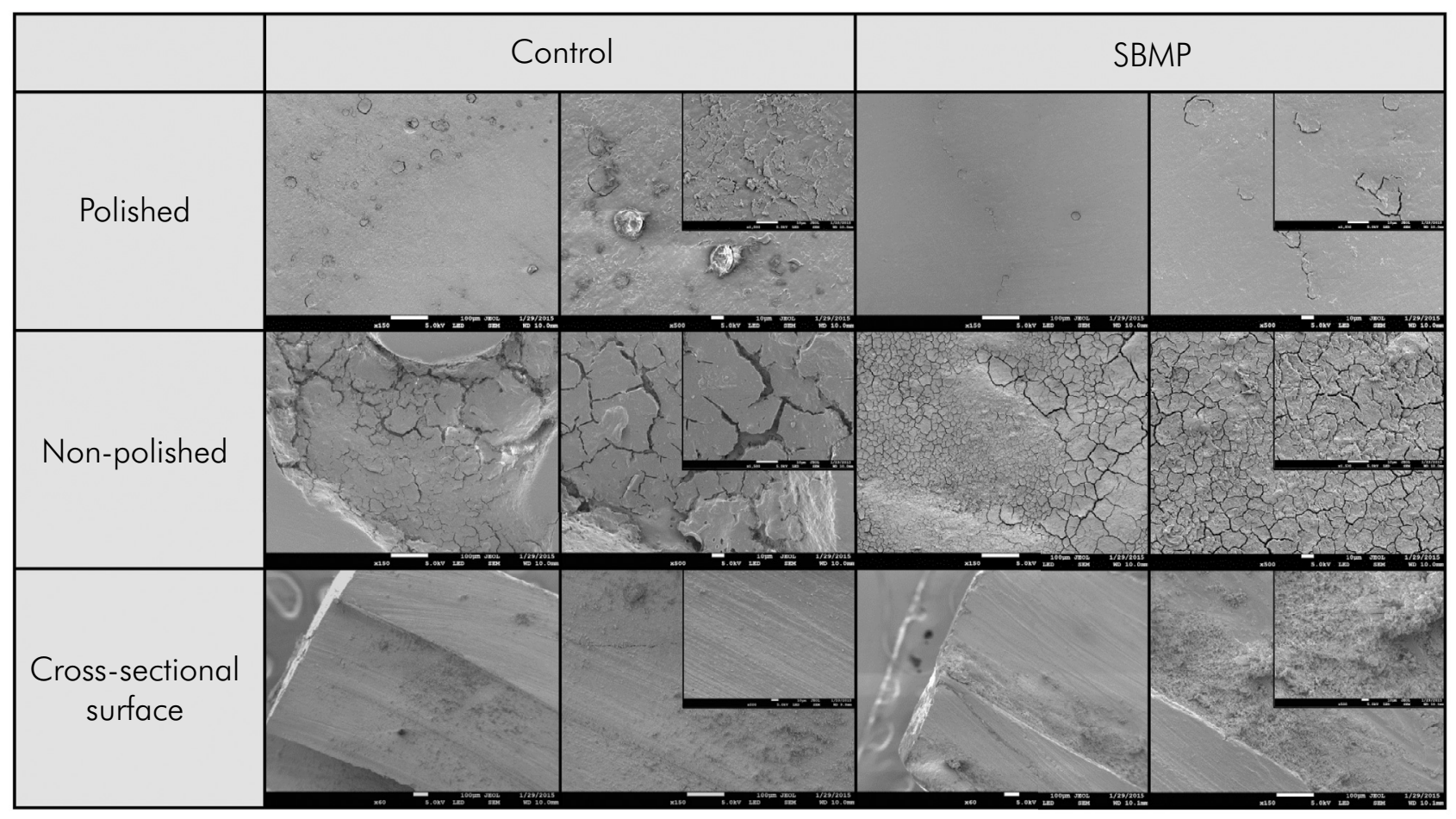

Figure 3. SEM images of the external and cross-sectional surfaces of the polished and non-polished specimens, with (SBMP) or without (control) modeling liquid, after 12 months of red wine storage.

with the application of a polishing paste that may produce a moderately impermeable surface, thus increasing the color stability of the material over time. ${ }^{26}$ Although the finishing/polishing procedure of composite restorations is a recommended clinical step in restorative treatment, an optimal finished/polished surface cannot be expected in all clinical circumstances, as there are some areas of the restoration, including proximal surfaces and posterior areas, which may be difficult to polish. This may lead to unavoidable color mismatch in the restorative material, as color change is more likely in non-polished surfaces than well-polished surfaces, as shown by the findings of the present study. In addition, the non-polished specimens permitted the formation of a crust complex over their surface (Figure 3), facilitating the binding of wine staining/molecules to the surface and possibly contributing to the intense color change observed in the non-polished specimens. Taking this into consideration, it may be that the surface of the material in specimens that are non-polished may remain un-polymerized (i.e., active) due to the oxygen inhibition effect, which renders the material susceptible to "glue" and to absorb stains from the environment. In addition, it is common for the finishing and polishing procedures of composite restorations to be performed during a subsequent clinical appointment, thus the presence of a rough surface on the restorative material, ${ }^{27}$ even for a short period of time, may lead to staining, as observed after one day of wine storage (data not shown), in which the non-polished specimens became more red in color, compared with their polished counterparts.

Despite both the SBMP and control groups exhibiting increased color change in the non-polished specimens, compared with the polished specimens, it is important to note that, within the non-polished specimens, the control group exhibited significantly higher discoloration than the SBMP group (Figure 1d and Table 1). It may be that the presence of the modeling liquid protected the composite against fast degradation/staining, without preventing this negative outcome. Furthermore, the visual perception of color change in restorations occurs when the $\Delta E^{*}$ value is $>3.3^{28}$ and considering that the $\Delta E^{*}$ values obtained in the present study were $>3.3$, regardless 
of the condition investigated, neither the presence of modeling liquid or a polished surface prevented discoloration of the material, although they were able to reduce severe staining.

On analysis of the SEM images (Figure 3), it was observed that, after the storage period, even the non-polished specimens prepared with the modeling liquid appeared smoother than those in the control group. It is known that the optical properties of dental RCs are directly affected by surface roughness, in the same way that an increasingly roughened surface reflects light differently, ${ }^{22}$ which leads to the perception of color change in the substrate. In addition, according to Bollen et al., ${ }^{29}$ the

\section{References}

1. Campodonico CE, Tantbirojn D, Olin PS, Versluis A. Cuspal deflection and depth of cure in resin-based composite restorations filled by using bulk, incremental and transtooth-illumination techniques. J Am Dent Assoc. 2011;142(10):1176-82. doi:10.14219/jada.archive.2011.0087

2. Frauscher KE, Ilie N. Depth of cure and mechanical properties of nano-hybrid resin-based composites with novel and conventional matrix formulation. Clin Oral Investig. 2012;16(5):1425-34. doi:10.1007/s00784-011-0647-3

3. Chang HS, Hong SO. Effects of layering technique on the shade of resin overlays and the microhardness of dual cure resin cement. Braz Oral Res. 2014;28(1):28. doi:10.1590/1807-3107BOR-2014.vol28.0016

4. Pontons-Melo JC, Furuse AY, Mondelli J. A direct composite resin stratification technique for restoration of the smile. Quintessence Int. 2011;42(3):205-11.

5. Eliades GC, Caputo AA. The strength of layering technique in visible light-cured composites. J Prosthet Dent. 1989;61(1):31-8. doi:10.1016/0022-3913(89)90104-2

6. Perdigão J, Gomes G. Effect of instrument lubricant on the cohesive strength of a hybrid resin composite. Quintessence Int. 2006;37(8):621-5.

7. Tjan AH, Glancy JF. Effects of four lubricants used during incremental insertion of two types of visible light-activated composites. J Prosthet Dent. 1988;60(2):189-94. doi:10.1016/0022-3913(88)90314-9

8. Barcellos DC, Pucci CR, Torres CR, Goto EH, Inocencio AC. Effects of resinous monomers used in restorative dental modeling on the cohesive strength of composite resin. J Adhes Dent. 2008;10(5):351-4

9. Tuncer S, Demirci M, Tiryaki M, Unlü N, Uysal Ö. The effect of a modeling resin and thermocycling on the surface hardness, roughness, and color of different threshold of surface roughness for biofilm formation is $0.2 \mu \mathrm{m}$ and, according to Aykent et al., ${ }^{26}$ surface roughness and bacterial adhesion are positively related, further supporting the requirement of an adequate finishing/polishing procedure for composite restorations.

\section{Conclusion}

The use of a modeling liquid, with a similar composition to that used in the present study, between layers of RC may reduce or delay the staining of a material over time. Moreover, the polishing of the material is essential to increase the color stability of the composite restoration.

resin composites. J Esthet Restor Dent. 2013;25(6):404-19. doi:10.1111/jerd.12063

10. Tanaka A, Nakajima M, Seki N, Foxton RM, Tagami J. The effect of tooth age on colour adjustment potential of resin composite restorations. J Dent. 2015;43(2):253-60. doi:10.1016/j.jdent.2014.09.007

11. Samra AP, Pereira SK, Delgado LC, Borges CP. Color stability evaluation of aesthetic restorative materials. Braz Oral Res. 2008;22(3):205-10. doi:10.1590/S1806-83242008000300003

12. Soares-Geraldo D, Scaramucci T, Steagall-Jr W, Braga SR, Sobral MA. Interaction between staining and degradation of a composite resin in contact with colored foods. Braz Oral Res. 2011;25(4):369-75. doi:10.1590/S1806-83242011000400015

13. Topcu FT, Sahinkesen G, Yamanel K, Erdemir U, Oktay EA, Ersahan S. Influence of different drinks on the colour stability of dental resin composites. Eur J Dent. 2009;3(1):50-6.

14. Sirin Karaarslan E, Bulbul M, Yildiz E, Secilmis A, Sari F, Usumez A. Effects of different polishing methods on color stability of resin composites after accelerated aging. Dent Mater J. 2013;32(1):58-67. doi:10.4012/dmj.2012-045

15. Kaizer MR, Diesel PG, Mallmann A, Jacques LB. Ageing of silorane-based and methacrylate-based composite resins: effects on translucency. J Dent. 2012;40 Suppl 1:e64-71. doi:10.1016/j.jdent.2012.04.014

16. Camargo FM, Della Bona A, Moraes RR, Coutinho de Souza CR, Schneider LF. Influence of viscosity and amine content on $\mathrm{C}==\mathrm{C}$ conversion and color stability of experimental composites. Dent Mater. 2015;31(5):e109-15. doi:10.1016/j.dental.2015.01.009 
17. Sarafianou A, Iosifidou S, Papadopoulos T, Eliades G. Color stability and degree of cure of direct composite restoratives after accelerated aging. Oper Dent. 2007;32(4):406-11. doi:10.2341/06-127

18. Barutcigil Ç, Yıldız M. Intrinsic and extrinsic discoloration of dimethacrylate and silorane based composites. J Dent. 2012;40 Suppl 1:e57-63. doi:10.1016/j.jdent.2011.12.017

19. Falkensammer F, Arnetzl GV, Wildburger A, Freudenthaler J. Color stability of different composite resin materials. J Prosthet Dent. 2013;109(6):378-83. doi:10.1016/S0022-3913(13)60323-6

20. Münchow EA, Sedrez-Porto JA, Piva E, Pereira-Cenci T, Cenci MS. Use of dental adhesives as modeler liquid of resin composites. Dent Mater. 2016 Feb 2. Pii: S0109-5641(16)00019-1. doi:10.1016/j.dental.2016.01.002

21. Uchimura JY, Sato F, Bianchi G, Baesso ML, Santana RG, Pascotto RC. Color stability over time of three resin-based restorative materials stored dry and in artificial saliva. J Esthet Restor Dent. 2014;26(4):279-87. doi:10.1111/jerd.12106

22. Sarac D, Sarac YS, Kulunk S, Ural C, Kulunk T. The effect of polishing techniques on the surface roughness and color change of composite resins. J Prosthet Dent. 2006;96(1):33-40. doi:10.1016/j.prosdent.2006.04.012

23. Barakah HM, Taher NM. Effect of polishing systems on stain susceptibility and surface roughness of nanocomposite resin material. J Prosthet Dent. 2014;112(3):625-31. doi:10.1016/j.prosdent.2013.12.007
24. Rodrigues-Junior SA, Chemin P, Piaia PP, Ferracane JL. Surface roughness and gloss of actual composites as polished with different polishing systems. Oper Dent. 2015;40(4):418-29. doi:10.2341/14-014L

25. Ereifej NS, Oweis YG, Eliades G. The effect of polishing technique on 3-D surface roughness and gloss of dental restorative resin composites. Oper Dent. 2013;38(1):E1-12. doi:10.2341/12-122-L

26. Aykent F, Yondem I, Ozyesil AG, Gunal SK, Avunduk MC, Ozkan S. Effect of different finishing techniques for restorative materials on surface roughness and bacterial adhesion. J Prosthet Dent. 2010;103(4):221-7. doi:10.1016/S0022-3913(10)60034-0

27. Venturini D, Cenci MS, Demarco FF, Camacho GB, Powers JM. Effect of polishing techniques and time on surface roughness, hardness and microleakage of resin composite restorations. Oper Dent. 2006;31(1):11-7. doi:10.2341/04-155

28. Schulze KA, Marshall SJ, Gansky SA, Marshall GW. Color stability and hardness in dental composites after accelerated aging. Dent Mater. 2003;19(7):612-9. doi:10.1016/S0109-5641(03)00003-4

29. Bollen CM, Lambrechts P, Quirynen M. Comparison of surface roughness of oral hard materials to the threshold surface roughness for bacterial plaque retention: a review of the literature. Dent Mater. 1997;13(4):258-69. doi:10.1016/S0109-5641(97)80038-3 